\title{
Situs inversus uniportal video-assisted thoracoscopic right anatomic segmentectomy S1-S2 and S6
}

\author{
Diego Gonzalez-Rivas ${ }^{1,2}$, Kostis Soultanis ${ }^{2}$, Hui Zheng ${ }^{2}$, Yuming Zhu ${ }^{2}$ \\ ${ }^{1}$ Department of Thoracic Surgery and Minimally Invasive Thoracic Surgery Unit (UCTMI), Coruña University Hospital, Coruña, Spain; \\ ${ }^{2}$ Department of Thoracic Surgery, Shanghai Pulmonary Hospital, Tongji University School of Medicine, Shanghai 200433, China \\ Correspondence to: Diego Gonzalez-Rivas. Department of thoracic surgery, Coruña University Hospital, Xubias 84, 15006 Coruña, Spain. \\ Email: diego.gonzalez.rivas@sergas.es.
}

\begin{abstract}
Situs inversus totalis (SIT) is an extremely rare condition where the major organs are reversed or mirrored from their usual positions. There are few reports in the literature describing thoracoscopic major surgery in patients with this unusual anomaly. Here we report a 48-year old female with two small early stage lung cancer lesions located in the apicoposterior segment of the right upper lobe and in the dorsal segment of the right lower lobe. The patient underwent a uniportal approach through a $3 \mathrm{~cm}$ incision and combined anatomic segmentectomies in the upper and lower lobes. The postoperative course was uneventful. This is the first report of an anatomic segmentectomy in a patient with a situs inversus condition.
\end{abstract}

Keywords: Situs inversus; anatomic segmentectomy; uniportal video-assisted thoracoscopic surgery (uniportal VATS); single port segmentectomy; lung cancer

Submitted Feb 26, 2018. Accepted for publication Apr 11, 2018.

doi: $10.21037 /$ jtd.2018.04.97

View this article at: http://dx.doi.org/10.21037/jtd.2018.04.97

\section{Introduction}

Situs inversus is a congenital condition in which the major organs are reversed or mirrored from their usual positions. It probably originates from an abnormal rotation of the cardiac tube during embryogenesis (1).

It is an autosomal recessive genetic condition. The prevalence of situs inversus varies among different populations but it is around 1 in 10,000 people. In this case the heart was swapped to the right side of the thorax which is known as situs inversus with dextrocardia or SIT. The association between situs inversus and Kartagener's syndrome which is characterized by the triad of situs inversus, chronic sinusitis, and bronchiectasis, is frequent. About $25 \%$ of individuals with situs inversus have an underlying condition known as primary ciliary dyskinesia which causes increased susceptibility to lung infections (2).

Many people with SIT are unaware of their atypical anatomy until they need a radiological examination.

\section{Clinical case}

A 48-year-old female non-smoker patient was admitted to our department for surgery. She was diagnosed with an SIT condition. A CT scan revealed two ground glass opacity (GGO) lesions: an $0.8 \mathrm{~cm}$ GGO lesion located in segment 6 of the lower lobe (Figure $1 A$ ) and a $1 \mathrm{~cm}$ GGO lesion located in S2 near the intersegmental plane with S1 (Figure 1B). The patient was proposed for uniportal video-assisted thoracoscopic surgery (VATS).

\section{Surgical treatment (Figure 2)}

The patient was placed in a left lateral decubitus position. A VATS approach through a single $3 \mathrm{~cm}$ incision was made in the right $4^{\text {th }}$ intercostal space. The right lung composition of two lobes, vascular and bronchial anatomy was exactly like a normal left lung. Aortic arch, aortopulmonary window, location of the heart and the subcarinal space were 

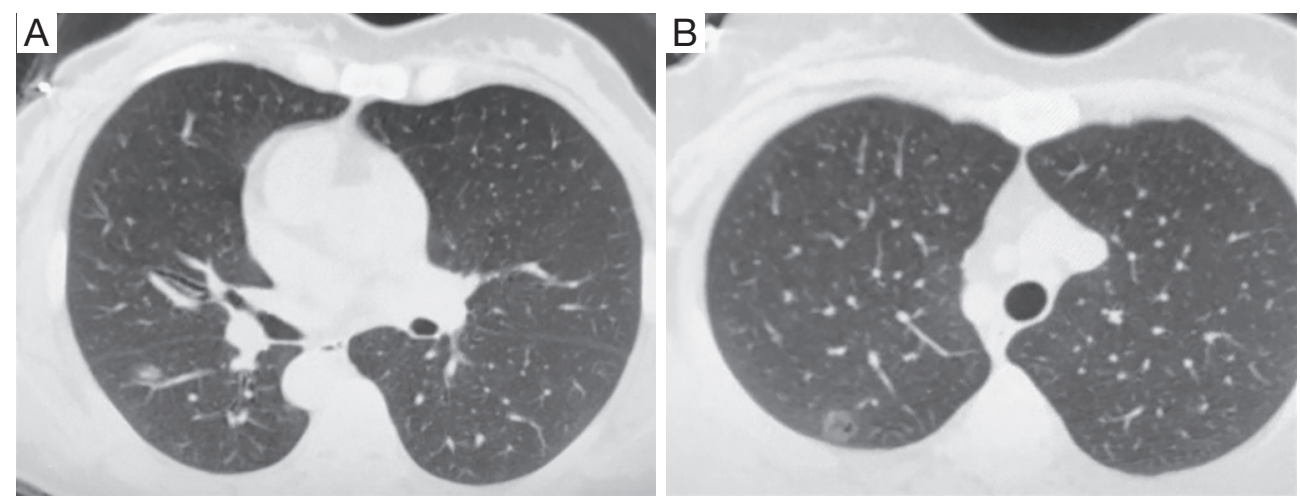

Figure 1 CT scan showing a $0.8 \mathrm{~cm}$ GGO tumor located in dorsal segment of right lower lobe (A) and another $1 \mathrm{~cm}$ GGO tumor located in the intersegmental plane of apical and posterior segment of right upper lobe (B). GGO, ground glass opacity.

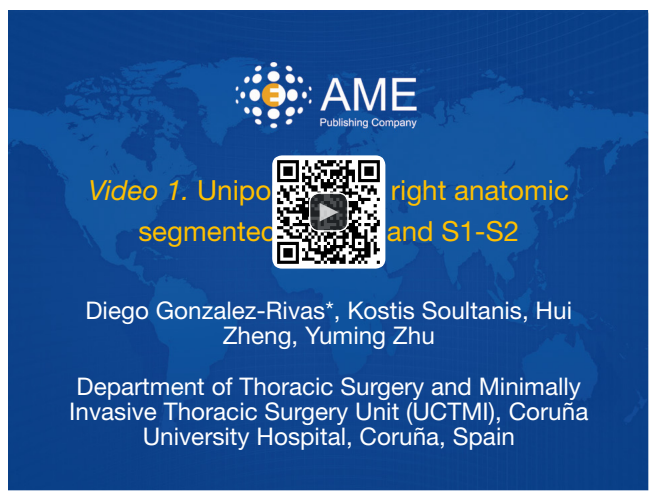

Figure 2 Uniportal VATS right anatomic segmentectomy S6 and S1-S2 (3). VATS, video-assisted thoracoscopic surgery.

Available online: http://www.asvide.com/article/view/24999

mirror images of a normal condition. We first performed an anatomic S6 segmentectomy (Figure $3 A$ ) and then an anatomic apicoposterior segmentectomy (S1-S2) was performed (Figure 3B). The identification of intersegmental plane was defined after the inflation-deflation technique, placing the staplers in between the proximal and distal stump of segmental bronchus. The distal S6 and S1S2 segmental stumps of vein, artery and bronchus were included in the resected anatomic segments.

The total surgical time was 55 minutes. The chest tube was removed on the second post-operative day and the patient was discharged on the fourth postoperative day. The final pathological examination revealed a minimally invasive adenocarcinoma (MIA) lesion on the lower lobe and adenocarcinoma in situ (AIS) lesion on the upper lobe.

\section{Discussion}

Most published situs inversus surgical reports are abdominal procedures and experience with SIT patients in minimally invasive thoracic surgery is limited. Cholecystectomy is the most frequent operation with SIT described in the literature (4). Cases of SIT in colorectal resections (5), esophageal (6), or cardiac procedures (7) have been reported. The initial publications of lung resections with SIT were described by thoracotomy: lung transplantation (8) and lung cancer surgery (9). The first cases of SIT in thoracoscopic lobectomy were reported in $2013(10,11)$. Since then a few groups have published cases on thoracoscopic lobectomy in patients with SIT, most of them by multi-portal approach (12). To our knowledge this is the first report of anatomic segmentectomy in a patient with an SIT condition.

Possible vascular or bronchial anomalies should always be taken into consideration during surgery on these patients. In this particular case, we found in the right side the exact mirrored anatomy of the left side for S6 and S1-S2. The left lung was composed of two lobes separated by a complete fissure, and the aortopulmonary window, subcarinal space, aorta, heart and esophagus were located in exactly the mirror-image position as in the left side.

Thanks to screening programs with low dose CT radiation, the detection of early stage of lung cancer is growing, especially the GGO lesions. The prognosis of small tumors with a GGO component is good with a limited pulmonary resection such as anatomic segmentectomy. Although wedge resections may be sufficient for curing small lung tumors with a GGO component, these lesions are difficult to palpate especially when the nodule is deeply located. Anatomical segmentectomy is preferred in such 

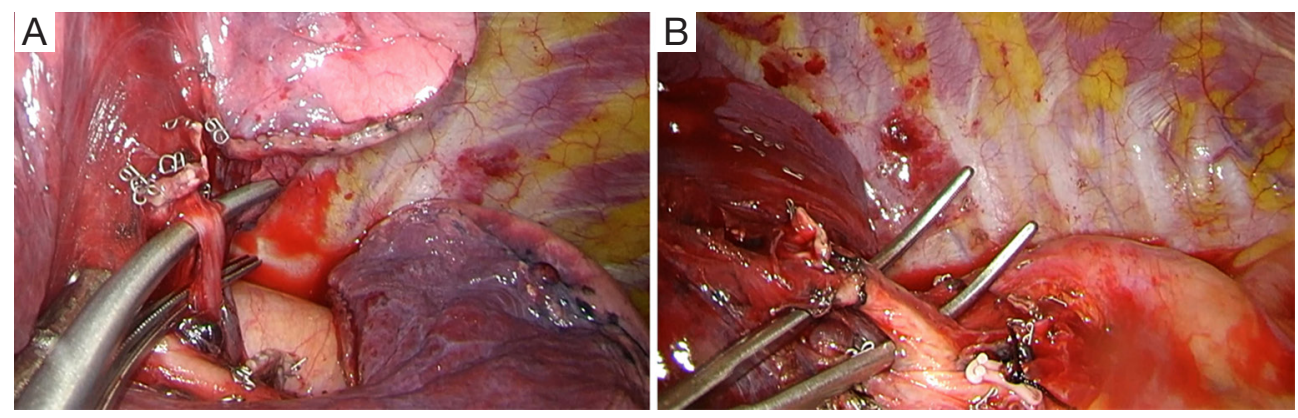

Figure 3 Thoracoscopic image of surgical dissection of V6 during dorsal segmentectomy (A) and apicoposterior bronchus during S1-S2 (B).

cases, both to secure an adequate surgical margin and remove distal peri-bronchial lymph nodes, and to preserve pulmonary function.

One of the most difficult steps during a segmentectomy is the identification and division of the intersegmental plane. There are several methods described in the literature. Stapling the demarcation line is our preferred method after inflation and deflation technique. However, the perfect knowledge of the segmental anatomy and the previous experience is mandatory to identify the correct intersegmental plane in order to perform a proper and oncologic anatomic resection.

The VATS anatomic segmentectomies are usually more demanding procedures than lobectomies, especially in a situs inversus condition. In these patients, the risk of vascular injuries or unexpected complications is higher due to the unusual anatomy.

In this particular case, the unusual orientation did not result in increased operative time, perhaps due to our previous experience in uniportal thoracoscopic lobectomies (13) and segmentectomies (14).

\section{Conclusions}

SIT is a challenging condition when surgery is necessary, especially when performing VATS major pulmonary resections. The uniportal thoracoscopic approach for anatomic segmentectomies is feasible in SIT patients but should be performed by skilled Uniportal VATS surgeons.

\section{Acknowledgements}

None.

\section{Footnote}

Conflicts of Interest: The authors have no conflicts of interest to declare.

Informed Consent: Written informed consent was obtained from the patient for publication of this case report and any accompanying images.

\section{References}

1. Subotich D, Mandarich D, Katchar V, Bulajich B, Drndarski B. Lung resection for primary bronchial carcinoma in a patient with complete situs inversus. Clin Anat 2006;19:358-62.

2. Teixeira J, Vaz A, Costa N. Bronchiectasis associated with total situs inversus and sinusitis: the Kartagener triad; case treated by lobectomy; review of literature. Rev Bras Cir 1952;23:431-42.

3. Gonzalez-Rivas D, Soultanis K, Zheng H, et al. Uniportal VATS right anatomic segmentectomy S6 and S1-S2. Asvide 2018;5:527. Available online: http://www.asvide. com/article/view/24999

4. Tacchino R, Greco F, Matera D. Single-incision laparoscopic cholecystectomy: surgery without a visible scar. Surg Endosc 2009;23:896-9.

5. Kim HJ, Choi GS, Park JS, et al. Laparoscopic right hemicolectomy with D3 lymph node dissection for a patient with situs inversus totalis: report of a case. Surg Today 2011;41:1538-42.

6. Yoshida T, Usui S, Inoue H, et al. The management of esophageal cancer with situs inversus totalis by simultaneous hand-assisted laparoscopic gastric mobilization and thoracoscopic esophagectomy. J 
Laparoendosc Adv Surg Tech A 2004;14:384-9.

7. Iino $\mathrm{K}$, Watanabe $\mathrm{G}$, Ishikawa $\mathrm{N}$, et al. Total endoscopic robotic atrial septal defect repair in a patient with dextrocardia and situs inversus totalis. Interact Cardiovasc Thorac Surg 2012;14:476-7.

8. Macchiarini P, Chapelier A, Vouhé P, et al. Double lung transplantation in situs inversus with Kartagener's syndrome. Paris-Sud University Lung Transplant Group. J Thorac Cardiovasc Surg 1994;108:86-91.

9. Murakawa T, Nakajima J, Fukami T, et al. Lung cancer operation in situs inversus totalis patient. Kyobu Geka 2009;62:1010-3.

10. Gonzalez-Rivas D, Fernandez Prado R, De la Torre M, et al. Situs Inversus Uniportal Video-Assisted Thoracoscopic
Left Middle Lobectomy. CTSNet, August 2013.

11. Yoshida M, Hino H, Machida H, et al. Video-assisted thoracic surgery lobectomy for lung cancer in a patient with complete situs inversus. Gen Thorac Cardiovasc Surg 2013;61:155-9.

12. Ye M, Shen J, Kong M, et al. Video-assisted thoracoscopic surgery right upper lobectomy in a situs inversus totalis patient. J Thorac Dis 2017;9:E1018-20.

13. Gonzalez-Rivas D, Paradela M, Fernandez R, et al. Uniportal video-assisted thoracoscopic lobectomy: two years of experience. Ann Thorac Surg 2013;95:426-32.

14. Gonzalez-Rivas D, Mendez L, Delgado M, et al. Uniportal video-assisted thoracoscopic anatomic segmentectomy. J Thorac Dis 2013;5 Suppl 3:S226-33.
Cite this article as: Gonzalez-Rivas D, Soultanis K, Zheng $\mathrm{H}, \mathrm{Zhu} \mathrm{Y}$. Situs inversus uniportal video-assisted thoracoscopic right anatomic segmentectomy S1-S2 and S6. J Thorac Dis 2018;10(5):E383-E386. doi: 10.21037/jtd.2018.04.97 